\title{
Impact of COVID-19 infections among kidney transplant recipients
}

\section{Dear Editor,}

More than 2 years since the start of the COVID-19 pandemic, cases continue to climb despite global efforts at viral control. This is largely driven by the emergence of viral variants. In the later part of 2021, the Delta variant was the predominant variant circulating globally, and was associated with increased infectivity, transmissibility, reduced vaccine response, and reduced susceptibility to monoclonal antibodies. ${ }^{1-3}$

In Singapore, despite having a robust test-trace-isolate strategy since the start of the pandemic, and one of the highest vaccination rates in the world with more than $80 \%$ of our population being fully vaccinated, the Delta variant has managed to "break through" our control measures, causing an exponential increase in the number of COVID-19 cases since the start of September 2021. ${ }^{4}$ In addition, as restrictions loosen for practical and economic reasons, it is inevitable that COVID-19 infections in the immunocompromised patients (including kidney transplant recipients) will increase. Prior to September 2021, there were no reported COVID-19 infections in kidney transplant recipients on our follow-up. However, within the first few weeks of lifting restrictions (1 September to 15 October 2021), 12 of our patients were infected. Among them, 10 were treated at Singapore General Hospital and are described in this report. We had existing approval by the ethics committee of the institution to evaluate longitudinal outcomes in kidney transplant.

Patient demographics, clinical presentation and outcomes are described in Table 1 (also Table S1 of Supplementary Materials in the online version of this article). Their median age was 61.5 (range 52-65) years. Most patients acquired COVID-19 from the community-6 (60\%) from close contacts (either household or social contacts), 1 (10\%) nosocomial transmission, and 3 (30\%) unlinked. Seven (70\%) patients had received at least 2 doses of the Pfizer BNT162b2 vaccine, and only $2(28.6 \%)$ of these 7 had a serological response. The median time to infection from the second dose of vaccine was 137 (39-164) days. Three $(30 \%)$ patients were unvaccinated, 1 of whom had prior COVID-19 infection in India.

Most patients $(80 \%)$ developed mild to moderate infections; the $2(20 \%)$ patients who developed severe infection required only supplementary oxygen therapy via nasal prongs. None developed graft dysfunction as defined by $>25 \%$ increase in baseline creatinine, and none were reinitiated on dialysis. Eight $(80 \%)$ patients were admitted within the first 7 days of illness, and received early COVID-19 specific therapeutics. Five $(50 \%)$ received sotrovimab monotherapy, $6(60 \%)$ remdesivir, $1(10 \%)$ remdesivir/dexamethasone, and $1(10 \%)$ received sotrovimab on admission, followed by remdesivir/dexamethasone when she developed type 1 respiratory failure. Patients who developed lymphopaenia and/or were symptomatic with moderate to severe disease had their anti-metabolite suspended promptly. Calcineurin inhibitors and mechanistic target of rapamycin (mTOR) inhibitors doses were maintained; patients with mild-to-moderate disease were maintained on their regular prednisolone doses.

Four $(40 \%)$ patients developed bacterial co-infections ( 2 urinary tract infections and 2 pneumonia). None developed cytomegalovirus reactivation. All our patients have recovered and are discharged. For de-isolation purposes, we repeated nasopharyngeal swabs for SARS-CoV-2 polymerase chain reaction (PCR) and de-isolated patients when the cycle threshold of $>30$ was achieved. None demonstrated viral rebound based on serial PCR testing. Therefore, the theoretical risk of new COVID-19 variants developing in our cohort with stable immunosuppression was deemed negligible. The median time to meet criteria for de-isolation is 21 (17-22) days.

In our series, there are several key observations.

Firstly, in spite of our best efforts to mitigate the risk of COVID-19 through multipronged approach by educating our patients on public health measures (universal masking, safe distancing and good hand hygiene) in April 2020; conducting webinars on the importance of COVID-19 vaccination in transplant recipients in February 2021; and rolling out a mass vaccination programme with 90\% uptake among 867 patients known to us, zero COVID-19 acquisition for our patients is impossible now that COVID-19 is endemic.

Secondly, consistent with published data, vaccine responses following a 2-dose mRNA COVID-19 vaccination series in our transplant recipients are poor, 


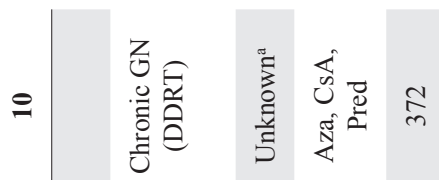

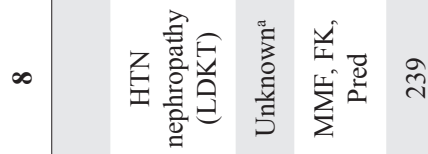

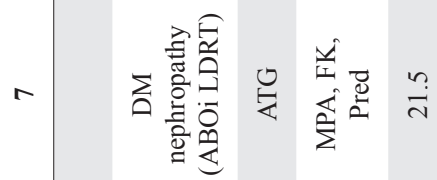

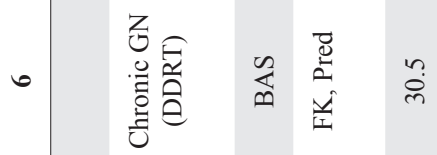

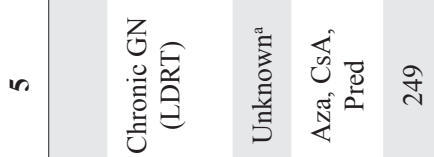

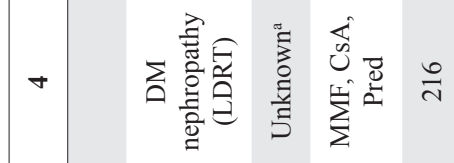

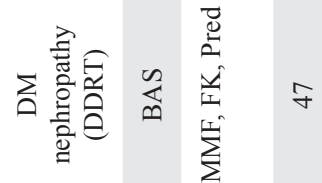

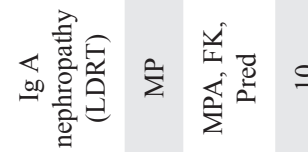

Z

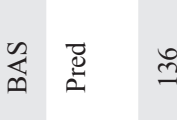

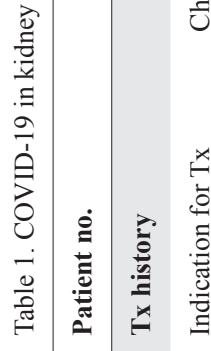

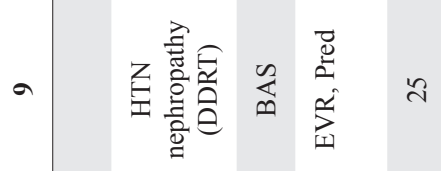

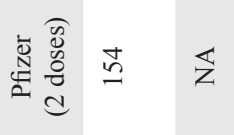

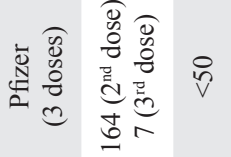

z $\quad \frac{\pi}{z}$

起兽

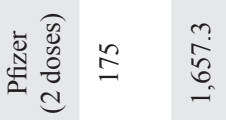

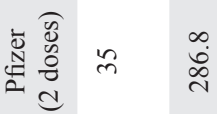

离高

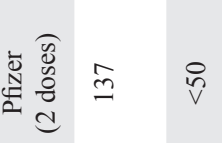

そั ฐ ถี

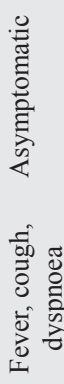

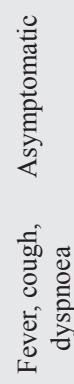

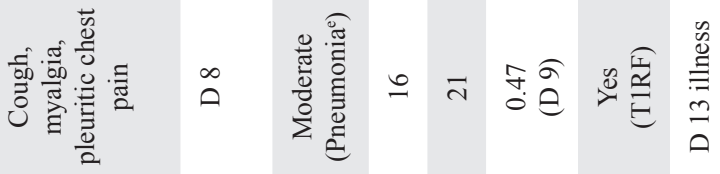

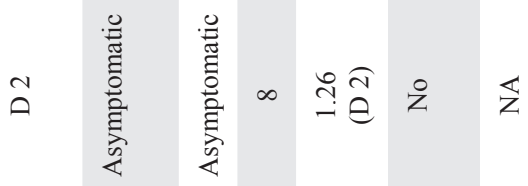

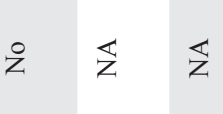

童言

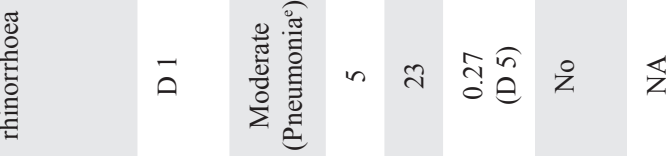

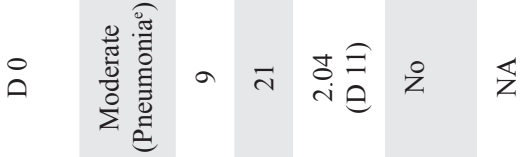

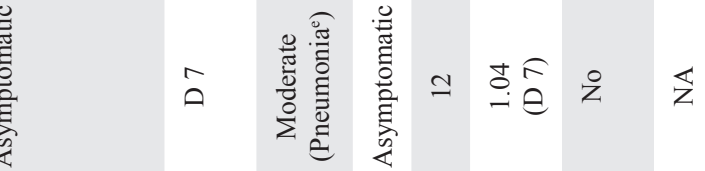

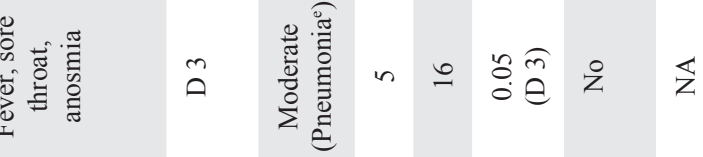

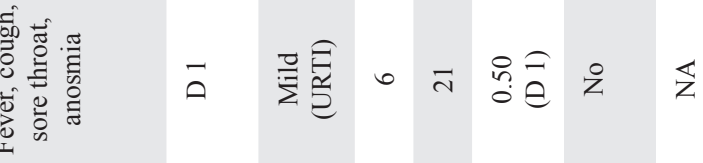

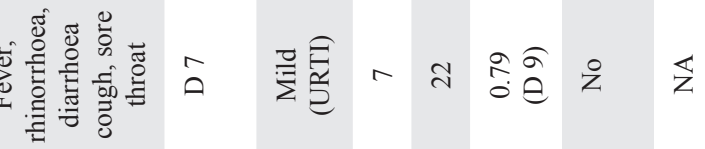

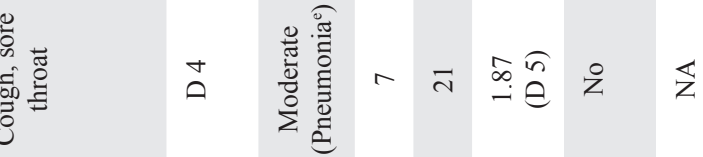

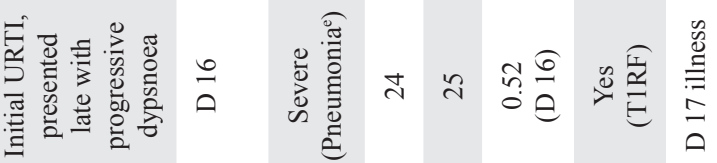
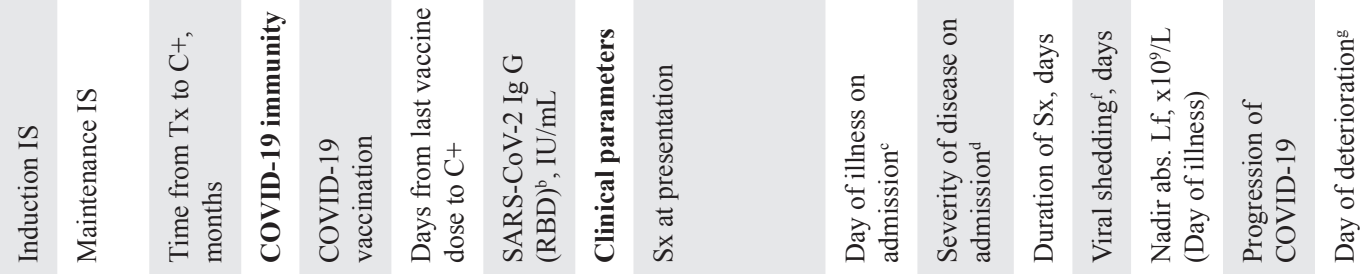


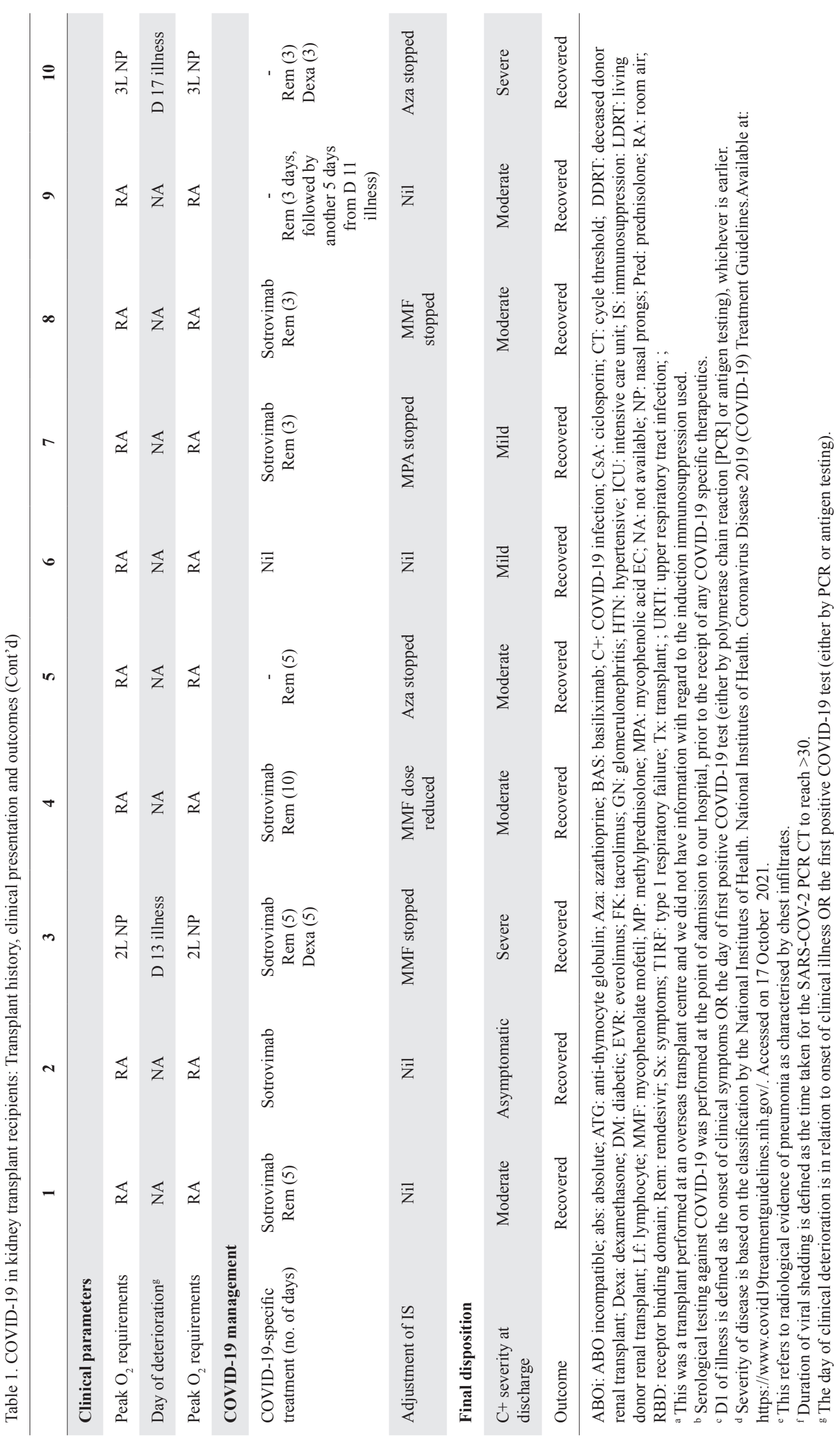


at only $28.6 \% .^{5}$ Most patients had SARS-CoV-2 immunoglobulin $\mathrm{G}$ receptor bonding domain level of $<50 \mathrm{IU} / \mathrm{mL}$. There was only 1 patient with antibody levels $>1,000 \mathrm{IU} / \mathrm{mL}$. Interestingly, his infection was mild, and required only symptomatic treatment. Intuitively, additional vaccine doses may improve vaccine responses, and this has also been published. With a third dose mRNA vaccine, serological responses improved significantly from $40 \%$ to $68 \%$; a 3-dose mRNA primary vaccination series (at a minimum) is now recommended for transplant recipients. ${ }^{6}$ Even so, post-vaccination seroprotective thresholds are not defined, and waning immunity postvaccination is increasingly being recognised. ${ }^{7,8}$ As such, routine serological testing post-vaccination in transplant recipients is not standard of care, and should not be routinely performed.

With regard to outcomes, most of our patients had mild-to-moderate infections. Only $2(20 \%)$ developed severe pneumonia but they never required critical care. This is in contrast to reported data from the earlier phases in the pandemic where approximately $40 \%$ of solid organ transplant recipients required intensive care. ${ }^{9}$ There are 2 possibilities. Perhaps a 2-dose mRNA COVID-19 vaccination series could still have induced T-cell responses (not assessed by serological assays) and this reduced the risk of progression to severe disease. ${ }^{5,10}$ However, our unvaccinated patients (albeit small numbers) also had relatively good outcomes. Therefore, a more likely reason is that we have adopted a more aggressive approach by admitting all known COVID-19 infected patients for consideration of monoclonal antibody therapy (in sero-negative patients, or those with relatively lower antibody titres) and early treatment with remdesivir where appropriate. ${ }^{11,12}$ This strategy is different from how we treat bacterial infections, where therapeutics are usually reserved for the sick or symptomatic. We would also like to highlight that even in mildly symptomatic COVID-19 infected transplant recipients, there was radiological evidence for pneumonia early in their illness, supporting the early administration of remdesivir. ${ }^{11}$ In addition, titration of immunosuppressants by our transplant physicians (in particular the early suspension of the anti-metabolites in patients who are lymphopaenic or those with progressive disease) was useful. ${ }^{11}$ We postulate that the efforts to prevent viral attachment and replication in our patients may have mitigated the downstream inflammatory responses seen in COVID-19. ${ }^{11,12}$
During the Delta outbreak, we recommend early evaluation and treatment of COVID-19 infected kidney transplant recipients in an inpatient setting, where they can receive early access to therapeutics (which are only available parenterally) and be closely monitored for complications. Moving forward, the model of care (e.g. management in acute hospital versus dedicated community care facility vs home recovery with telemedicine support) depends on several factors: (1) infectivity and virulence of the circulating SARS-CoV-2 virus, (2) medical complexity of the patients, (3) vaccine efficacy in solid organ transplant recipients, (4) access to approved therapeutics, (5) prevailing COVID-19 infection prevention goals and strategies, (6) capacity of our healthcare systems (including hospital bed availability, manpower availability, infrastructure, and auxiliary support for community care and telemedicine) and (7) psychological-social-cultural expectations of Singapore residents. We will have to continually modify our strategies based on pandemic situation, available resources, and emerging scientific data that inform care.

Finally, an ounce of prevention is worth a pound of cure. Public health measures, and vaccination of our patients and their close contacts, remain key pillars of the global COVID-19 control strategy.

\section{Acknowledgements}

We would like to thank all the medical, nursing and allied health staff who are working tirelessly in the isolation wards. Special mention to the crisis, planning and operations team who went out of their way to help us get our patients admitted. We would also like to thank Dr Limin Wijaya for reviewing the manuscript.

\section{REFERENCES}

1. GISAID. Tracking of Variants. Available at: https://www.gisaid.org/ hcov19-variants/. Accessed on 17 October 2021.

2. World Health Organization. Weekly epidemiological update on COVID-19 - 13 October 2021, 13 October 2021. Available at: https://www.who.int/publications/m/item/weekly-epidemiologicalupdate-on-covid-19---13-october-2021. Accessed on 19 October 2021.

3. Ong SWX, Chiew CJ, Ang LW, et al. Clinical and virological features of SARS-CoV-2 variants of concern: a retrospective cohort study comparing B.1.1.7 (Alpha), B.1.315 (Beta), and B.1.617.2 (Delta). Clin Infect Dis 2021:ciab721.

4. Ministry of Health, Singapore. COVID-19 Situation Report. Available at: https://covidsitrep.moh.gov.sg. Accessed on 18 October 2021.

5. Boyarsky BJ, Werbel WA, Avery RK, et al. Antibody Response to 2-Dose SARS-CoV-2 mRNA Vaccine Series in Solid Organ Transplant Recipients. JAMA 2021;325:2204-6. 
6. Kamar N, Abravanel F, Marion O, et al. Three Doses of an mRNA Covid-19 Vaccine in Solid-Organ Transplant Recipients. N Engl J Med 2021;385:661-2.

7. Ison MG, Blumberg E, Halasa N, et al. Antibodies, boosters, and optimizing SARS-CoV-2 vaccines for transplantation: A call for more research. Am J Transplant 2021.

8. Goldberg Y, Mandel M, Bar-On YM, et al. Waning Immunity after the BNT162b2 Vaccine in Israel. N Engl J Med 2021;385:e85.

9. Pereira MR, Mohan S, Cohen DJ, et al. COVID-19 in solid organ transplant recipients: Initial report from the US epicenter. Am J Transplant 2020;20:1800-8.

10. Aslam S, Adler E, Mekeel K, et al. Clinical effectiveness of COVID-19 vaccination in solid organ transplant recipients. Transpl Infect Dis 2021:e13705.

11. National Institutes of Health. Coronavirus Disease 2019 (COVID-19) Treatment Guidelines.Available at: https://www. covid19treatmentguidelines.nih.gov/. Accessed on 17 October 2021.

12. Dhand A, Lobo SA, Wolfe K, et al. Casirivimab-imdevimab for Treatment of COVID-19 in Solid Organ Transplant Recipients: An Early Experience. Transplantation 2021;105:e68-9.
Shimin Jasmine Chung ${ }^{1,3}{ }_{F R C P}$ (Edin),

Quan Yao $\underline{\text { Ho }}^{2,3}$ FAMS, Ian Tatt Liew ${ }^{2,3}$ MMed (S'pore),

Siew Yee Thien ${ }^{1}$ MRCP,

Yvonne Fu Zi Chan ${ }^{1}$ MRCP,

Benjamin Pei Zhi Cherng ${ }^{1}$ MMed,

Hei Man Wong ${ }^{1}$ MMed (S'pore), Ying Ying Chua ${ }^{1}$ MMed,

Terence $\underline{\mathrm{Kee}}^{2,3}{ }_{\text {FRCP }}$, Thuan Tong $\underline{\text { Tan }}{ }^{1,3}{ }_{\text {MRCP (UK) }}$

${ }^{1}$ Department of Infectious Diseases, Singapore General Hospital, Singapore

${ }^{2}$ Department of Renal Medicine, Singapore General Hospital, Singapore

${ }^{3}$ SingHealth Duke-NUS Transplant Centre, Singapore

Correspondence: Dr Shimin Jasmine Chung, Department of Infectious Diseases, Level 3, The Academia, Singapore General Hospital, 20 College Road, Singapore 169865.

Email: jasmine.chung.s.m@singhealth.com.sg 